In Crescendo, 2019; 10(4): 635-650

Fecha de recepción: 05/06/2019

Fecha de aceptación: 18/12/2019

\title{
BARRERAS EN LA IMPLEMENTACIÓN DE LA GAMIFICACIÓN EN EDUCACIÓN SUPERIOR: REVISIÓN DE LITERATURA
}

\author{
BARRIERS TO THE IMPLEMENTATION OF GAMIFICATION \\ IN HIGHER EDUCATION: LITERATURE REVIEW
}

\author{
Lira Isis Valencia-Quecano ${ }^{1}$, \\ David Orellana-Viñambres ${ }^{1}$
}

\section{RESUMEN}

El uso de gamificación en las principales instituciones de educación superior ha ido en aumento, los profesores pueden utilizarla para mejorar sus técnicas de enseñanza y aprendizaje y, sobre todo, para optimizar los escenarios de aprendizaje. Así, el principal objetivo de este estudio es identificar las barreras subyacentes en la implementación de estrategias didácticas gamificadas en educación superior, que permitan una mayor comprensión en su aplicación y utilización que posibiliten acciones de mejora al momento de utilizar la gamificación como un recurso pedagógico. La metodología utilizada se basa en un enfoque sistematizado compuesto de cinco fases en el desarrollo investigativo a saber: búsqueda, evaluación, análisis, síntesis y presentación. Como resultado final se obtuvieron 101 artículos que sirvieron como insumo para la identificación de cinco barreras (tecnología, diseño de la estrategia gamificada, pedagogía, docente y estudiante) y 29 barreras específicas en la implementación de estrategias didácticas gamificadas en el nivel de la educación superior. En conclusión, se puede inferir la importancia de los procesos de capacitación y cualificación docente en competencias tecnológicas que posibilitan una adecuada aplicación de la gamificación por los docentes y el resto de los profesionales en su propio contexto de aprendizaje virtual, así como la comprensión holística a partir de las más recientes perspectivas sobre gamificación. Finalmente, se sugiere realizar investigaciones que permitan la identificación de nuevas barreras en países de origen y modalidades de edu-

1 Corporación Universitaria de Asturias. 
cación, así como la obtención de datos especializados o del análisis de contenidos gamificados que complementen próximos estudios.

PALABRAS ClAVE: Barrera, Educación Superior, Gamificación, Estrategia de Aprendizaje y Enseñanza.

\section{ABSTRACT}

The gamification is increasing mostly in the main higher education institutions because teachers can use it to improve their teaching and learning skills and, over all, to optimize the learning scenarios. In this aspect, the main objective of this study is to identify the barriers in the implementation of gamified didactic strategies in higher education, for the purpose of a greater understanding of these factors, enabling improvement techniques when using gamification as a pedagogical resource. As methodology, a systematized approach is presented for the analysis of research articles that are related to these barriers in the use of gamification resources in higher education. Five phases are established: search, evaluation, analysis, synthesis and presentation. As results, 101 articles were obtained as input for the identification of five barriers (Technology, Gamified Strategy Design, Pedagogy, Teacher and Student) and 29 specific barriers in the implementation of gamified didactic strategies in higher education. In sum, it is important the qualification in technological competencies in education that enable teachers and other professionals to include adequately gamification in their own online learning context, as well as the development of a holistic understanding from the most recent gamification perspectives. Finally, it is suggested to do the identification of barriers considering the countries of origin and education modalities, as well as obtaining specialized data or the analysis of gamified contents that allows the identification of new barriers complementing future studies.

KEY WORDS: Barrier, Higher Education, Gamification, Learning and Teaching Skill.

\section{INTRODUCCIÓN}

El juego como expresión cultural, se ha caracterizado por facilitar el aprendizaje de códigos de la semiótica de la sociedad como lo expresa Huizinga (citado por Contreras, 2016), logrando incursionar en diversos entornos humanos. Por tanto, el concepto de gamificación se entiende como el uso de estrategias, dinámicas, mecánicas y elementos propios de los juegos, en contextos ajenos a estos, con la finalidad de transmitir contenidos a través de una experiencia lúdica (Llorens et al., 2016). De acuerdo con lo anterior, el campo de la educación ha sido uno de los entornos más propensos al uso de la gamificación, de tal manera que se teoriza sobre el aprendizaje basado en juegos, como una metodología que apoya el proceso de enseñanza-aprendizaje, a partir de la aplicación de las características de los juegos (Contreras, 2016). 
En este sentido, los juegos educativos se han venido incrementando en un 7.8\% (Entertamment Software Association, 2018), y es el nivel de la educación superior el de mayor aplicación de la gamificación, dado que, en las universidades, los docentes poseen una mayor libertad al diseñar sus clases, logrando la incorporación de estrategias que involucran elementos del juego (Oxford Analytica, 2016).

En la actualidad, la producción investigativa sobre gamificación en escenarios de educación superior se centra en describir el diseño de productos gamificados y su aplicación (Ding, 2019), establecer modelos para la creación de estrategias didácticas gamificadas (Mora et al., 2017); analizar el impacto de su implementación en diversas disciplinas académicas como la matemática, las ciencias sociales, las ciencias de la salud, y las ingenierías, entre otras (Gallegos et al., 2017).

También se relaciona la gamificación con el desarrollo cognitivo (Hernández et al., 2018); la emoción (Pérez et al., 2017); el fortalecimiento de habilidades sociales (Calabor et al., 2018) y el desarrollo de competencias específicas en diversas disciplinas académicas (Gutiérrez et al., 2018). De igual manera, se encuentran estudios que buscan analizar las percepciones de los estudiantes que interactúan con productos gamificados (Aldemir et al., 2018); la identificación de competencias tecnológicas docentes en el diseño e implementación de la gamificación en el aula (Kopcha et al., 2016) y, su percepción en cuanto al uso pedagógico de estrategias gamificadas en el salón de clase (Adukaite et al., 2017).

Teniendo en cuenta el panorama anterior, el presente documento busca identificar posibles obstáculos en la implementación de la gamificación en la educación superior, lo que permite resaltar su contribución teórica al generar un entendimiento mayor sobre la creación y aspectos de mejora en la aplicación de productos gamificados.

\section{METODOLOGÍA}

La presente investigación presenta un enfoque sistematizado (Grant y Booth, 2009), para el análisis de la literatura concerniente a la gamificación en escenarios de educación superior, durante el periodo de tiempo comprendido entre el año 2009 y el año 2019. Para su desarrollo, se establecieron cinco fases que se describen a continuación:

En la fase de búsqueda de información, se identificaron las palabras clave tanto en español como en inglés, tales como: "gamificación" OR "gamification", "gamifi- 
cación" AND educación AND barreras en gamificación" OR "gamification AND education AND barriers in gamification"; se complementó, de manera adicional, con términos como "problemas" OR "problems", "desafíos" OR "challenges", "obstáculos” OR "obstacles”, entre otros. Igualmente se aplicó como delimitación, el escenario de la educación superior. No se tuvo en cuenta, para esta investigación, criterios relacionados con el país de estudio ni la modalidad de educación.

En la fase de evaluación, se realizó la revisión y evaluación sistematizada de las bases de datos relevantes en el área de investigación, dado el impacto de las revistas indexadas, alojadas en ellas tales como SCOPUS Y Web Of Science (en adelante WOS), obteniendo un total de 489 artículos científicos. Se identificó que 185 trabajos no abordaban la gamificación en escenarios educativos, 59 documentos no tocaban el tema de gamificación en niveles de la educación superior y 65 escritos presentaron la característica de pertenecer a revistas Q3 y Q4 con menos de diez citas. Finalmente se retiraron 79 documentos que no mencionan alguna a las barreras que se presentan en la implementación de la gamificación en ámbitos de la educación superior obteniendo como resultado final, 101 documentos que aportan datos relevantes sobre las barreras para su implementación en el nivel de la educación superior.

A continuación, en la fase de análisis, se realizó un enfoque inductivo del contenido de los 101 artículos. Para compendiar los datos, en esta fase se elaboró una matriz que contiene los siguientes datos: nombre de los autores, año de la publicación, título del artículo, nombre de la revista, número y volumen, URL o DOI, objetivos, metodología abordada, resultados y conclusiones.

En la fase síntesis, se elaboró una segunda matriz que permite la identificación de barreras, barreras específicas, autores y observaciones. Finalmente, en la fase de presentación, se elaboró un diagrama (Véase Figura 4) que indica las barreras y las barreras específicas identificadas y clasificadas como tales. Y, en la tabla 1, se puntualiza el tipo de barrera, el tipo de barrera específica, los autores que las abordan y la descripción.

\section{RESULTADOS}

\section{Análisis de las publicaciones}

La revisión de los 101 artículos de investigación arrojó como resultado 26 países de origen de los escritos, los cuales se consolidan, en donde: Estados Unidos con el 29\% de artículos, seguido de España con el 18\%, China con el 9\% y 
Reino Unido con el 9\% de publicaciones de literatura investigativa en temas de gamificación aplicada a la educación superior.

Respecto a la distribución de los artículos de investigación por año en el periodo comprendido entre 2009 y 2019, el incremento del número de publicaciones se sitúa en el periodo 2015-2018, lo que indica un aumento en el interés de docentes e investigadores por comprender la incidencia de la gamificación en los procesos de enseñanza-aprendizaje suscitados en la educación superior.

Finalmente, la distribución por revista científica indica que, de las 56 revistas consultadas, el 23\% de estudios realizados fueron publicados por Computers and Education, seguido por la revista Computer in Human Behavior con una participación del 10\%, y en un tercer lugar se ubican las revistas Electronic Journal of E Learning, International Journal of Educational Technology in Higher Education y Medical Education con un porcentaje del 3\% cada una. En la categoría Otras Revistas, se encuentran 51 revistas, con una única publicación en el tema de gamificación en educación superior, que representa el $58 \%$ de la participación porcentual.

\section{Identificación y Categorización de Barreras}

Los trabajos de investigación sobre gamificación educativa, actualmente, se centran de manera fundamental, en temas como el diseño de estrategias gamificadas y su implementación en el aula (Aldemir et al., 2018); otros estudios han realizado revisiones documentales, en donde se destacan las ventajas en la utilización de la gamificación, haciendo énfasis en su relación con el aprendizaje, la motivación y la adquisición de competencias específicas (Buckley y Doyle, 2016). En cuanto a la identificación de barreras o desafíos, únicamente se encuentra el trabajo empírico realizado por Sánchez-Mena, y Martí-Parreño, (2017) quienes identificaron, a partir del análisis de 16 entrevistas realizadas a docentes, las percepciones sobre el uso de gamificación en el aula, categorizando cuatro facilitadores: atención-motivación, entretenimiento, interactividad y facilidad para aprender y cuatro barreras generales siendo estas: falta de recursos, apatía de los estudiantes, ajuste de la asignatura y dinámica del aula y nueve barreras específicas a saber: falta de tiempo, falta de capacitación, inapropiada infraestructura de clase, falta de apoyo económico, percepción de pérdida de tiempo, no alineación con las características del sujeto, no alineación con el horario de la asignatura, disturbios en el salón y finalmente conflicto con otros maestros. Sin embargo, el estudio de Sánchez-Mena y Martí-Parreño (2017), presentó como limitaciones 
investigativas, primero, un posible sesgo debido al tamaño de la muestra y a las edades de los sujetos, ya que se considera que no fue representativa de las características de la población objetivo y, segundo que la mayoría de los docentes entrevistados pertenecieran al área de mercadeo y comercio, limitando la generalización de los resultados.

Teniendo en cuenta lo anterior, la presente investigación buscó, identificar y categorizar las barreras en el uso de la gamificación en educación superior, desde una visión holística que involucra aspectos de la calidad educativa, a partir de la revisión de la literatura previamente seleccionada, encontrando limitaciones en los diferentes factores que componen el ejercicio de enseñanza-aprendizaje. En la literatura se encontraron cinco grandes barreras, que son la Tecnología, el Diseño de la Estrategia Gamificada, la Pedagogía, el Docente y el Estudiante, y, de manera específica, el reconocimiento de 29 barreras específicas sobre el tema en cuestión.

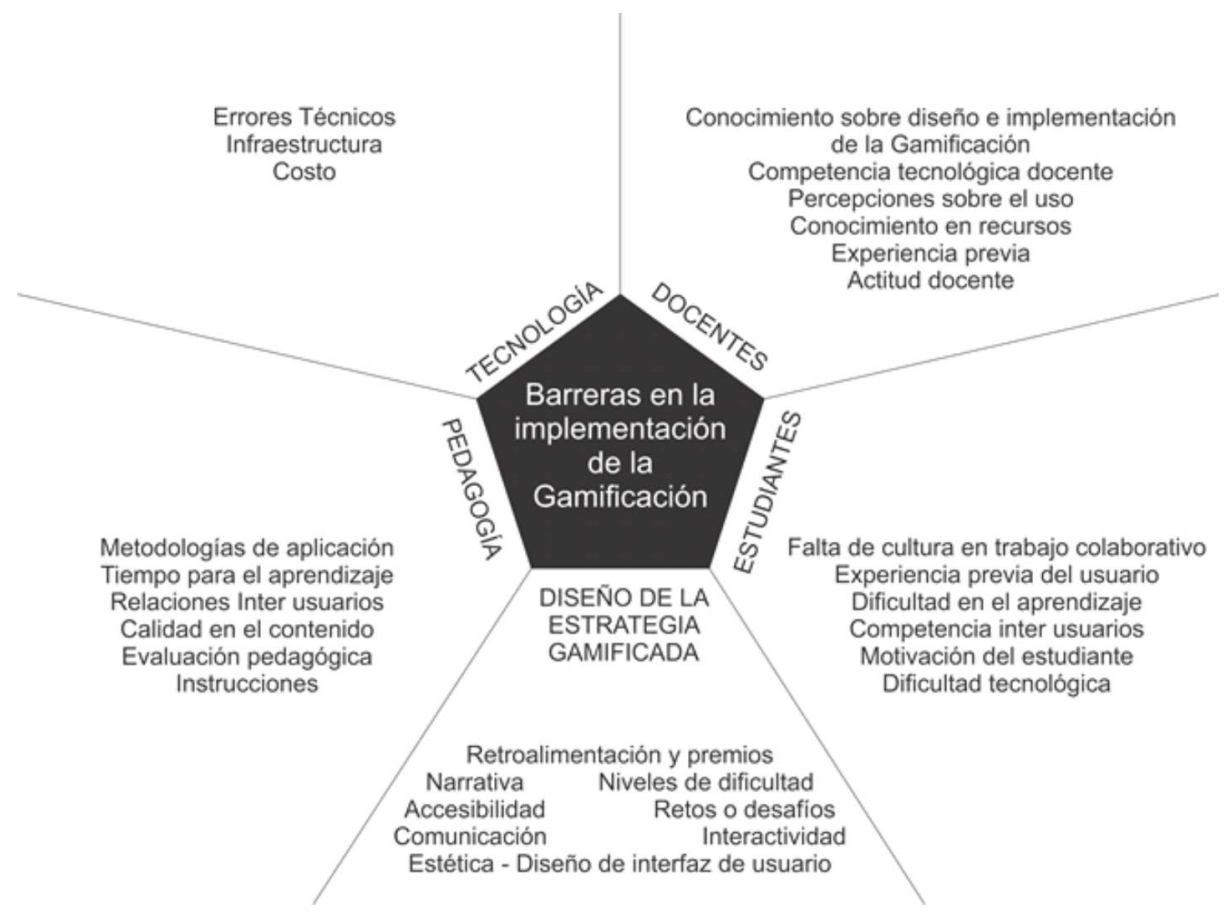

Figura 1. Barreras en la implementación de gamificación en entornos educativos. 
Con este gráfico se puede comprender un mayor alcance y una mayor extensión en la categorización de las barreras en la implementación de la gamificación en el aula, respecto de la clasificación propuesta por Sánchez-Mena y MartíParreño (2017).

\section{Barrera Tecnología}

En la barrera Tecnología, se encontraron tres barreras específicas, la primera Infraestructura se refiere a la insuficiencia en la cantidad de dispositivos para lograr que la totalidad de estudiantes puedan interactuar con la estrategia gamificada, esto se traduce en la falta de computadores o dispositivos tecnológicos alternos como celulares o tablets (Piñeiro y Costa, 2015). También relaciona problemas con la caducidad de las versiones gratuitas de ciertos juegos en línea o plataformas gamificadas para educación y la disminución en el acceso a internet, lo que lleva al uso de redes domésticas o tecnologías obsoletas (Adukeite et al., 2017).

Los Errores Técnicos encontrados en el estudio, hacen referencia a problemas relacionados con la programación de la estrategia gamificada, como la distorsión visual del contenido (pixelación); la pérdida de información del desempeño en el juego; fallas en la compatibilidad entre el hardware y software (Barr, 2017), así como falta de mantenimiento de los dispositivos al momento de la implementación de la estrategia gamificada (Ding, 2019). Por último, el Costo, es una barrera que se refiere al valor económico que debe ser pagado al programador o desarrollador al momento de diseñar, implementar y mantener la estrategia didáctica gamificada, así como a las tecnologías en hardware y software relacionadas con ella (Poondej y Lerdpornkulrat, 2016).

\section{Barrera Diseño de la Estrategia Gamificada}

La barrera Diseño de la Estrategia Gamificada, identifica ocho barreras específicas a saber: Estética y Diseño de Interfaz de Usuario, Narrativa, Niveles de Dificultad, Retroalimentación y Premios, Accesibilidad, Retos o Desafíos, Comunicación e Interactividad. La Estética y el Diseño de Interfaz de Usuario atañe al uso de diseños complejos que fomentan la distracción del objetivo educativo (Chang et al., 2017), el uso de diseños serios que no gustan al estudiante o el uso de marcos poco claros conceptualmente, que generan confusión al interior del juego (Piñeiro y Costa, 2015). La barrera Narrativa concierne a la falta de líneas lógicas en el guion establecido o en la narración concreta de la historia del juego 
(Aşıksoy, 2018). Algunos estudios manifiestan el factor de extensión en el texto, dado que puede propiciar carga cognitiva en los estudiantes (Blevins et al. 2017).

La barrera específica Niveles de Dificultad, hace referencia a los grados de complejidad en la resolución de los desafíos, retos o actividades propuestas, matizado en la existencia de retos que pueden ser simples o limitados en contraposición a desafíos que son difíciles. El nivel de complejidad debe obedecer a las características de la población objetivo a la cual se dirige, a fin de que los estudiantes logren dar solución a las actividades de la gamificación (Chen et al., 2015).

La Retroalimentación y Premios, como barrera, se refiere a la falta de relación entre la realimentación y el contenido pedagógico; la incoherencia entre estos dos elementos, fomenta la baja motivación frente a la estrategia didáctica gamificada (Acosta-Gonzaga y Walet, 2018), agregado a que al no poder consultar las tablas de clasificación (tablas de retroalimentación del desempeño del estudiante) en tiempo real, propicia una autopercepción de que no se es competitivo frente a los demás estudiantes (Poondej y Lerdpornkulrat, 2016). El elemento frecuencia, también debe ser tenido en cuenta dado que la escasa retroalimentación propicia la disminución de los comportamientos adecuados que deben ser emitidos por los estudiantes como parte del ejercicio de enseñanza-aprendizaje (White y Shellenbarger, 2018).

La limitación relacionada con la Accesibilidad hace referencia a la tendencia de presentar diseños o contenidos dirigidos a personas sin diversidad funcional, lo que dificulta a personas bajo esta condición, acceder a la estrategia gamificada. Asimismo, el omitir las diversas características culturales y psicológicas de los estudiantes, puede generar obstáculos al momento de acceder al contenido gamificado (Adukaite et al., 2017).

También elementos como los Retos o Desafíos, si son sencillos, pueden impactar la motivación del estudiante y alejarlo del contenido gamificado. Lo mismo ocurre cuando los retos requieren de esfuerzos elevados para conseguir el premio o los puntos (Auvinen et al., 2015). La Comunicación, como limitación refiere a la ausencia de espacios para la interacción social, al disminuir las oportunidades para el desarrollo de la colaboración entre pares, situación que decrementa la posibilidad de aprender en conjunto (de Freitas y de Freitas, 2013).

Finalmente, la barrera específica Interactividad, se presenta cuando el nivel de interacción se polariza a niveles bajos o altos para alcanzar el objetivo y depende de las destrezas del estudiante, por ello la interactividad guarda relación con las características de la población objetivo (Wang et al., 2011). 


\section{Barrera Pedagogía}

La barrera relacionada con la Pedagogía permitió reconocer seis barreras específicas, así: Calidad en el Contenido, Metodologías de Aplicación, Evaluación Pedagógica, Relaciones Inter-usuarios, Tiempo para el Aprendizaje, e Instrucciones. Dentro de estas seis barreras específicas la Calidad en el Contenido refiere a la coherencia y cohesión existente en el diseño instruccional del producto gamificado, lo que hace que dicho contenido sea lo suficientemente claro y lógicamente organizado para la obtención de los objetivos del aprendizaje de parte del estudiante (Piñeiro y Costa, 2015). También algunas obras reportan la existencia de estrategias gamificadas que llevan a la confusión al ubicar temas que no guardan relación (Buckley y Doyle, 2017), así como la falta de consistencia entre los elementos propios de la pedagogía y los concernientes al diseño del juego los cuales generan niveles altos de frustración, lo que a corto y mediano plazo propicia situaciones de deserción estudiantil (Alhammad y Moreno, 2018) $\mathrm{o}$, la ausencia de mecanismos que permitan al estudiante comprender las relaciones entre conceptos (Montes-González et al., 2018). En este aspecto es importante tener en cuenta el manejo ético inmerso en el diseño e implementación de estrategias didácticas gamificadas, respetando las características personales de los estudiantes, a fin de evitar contenidos inadecuados relacionados con la edad o con la cultura.

Las Metodologías de Aplicación, como barrera específica, se relacionan con la falta de documentación sobre técnicas claras en temas de implementación de estrategias gamificadas en el aula, lo que genera vacíos pedagógicos en relación con la claridad procedimental al momento de la aplicación (Wiggins, 2016). La Evaluación Pedagógica, también se identifica como barrera, cuando no se establecen los modelos para realizar la adecuada evaluación del aprendizaje adquirido por el estudiante mediante el uso de la gamificación (Dicheva et al., 2015).

En lo que respecta a las Relaciones Inter usuarios, los productos gamificados pueden variar de acuerdo a las posibilidades de interacción entre los estudiantes; así pueden existir juegos individuales o juegos que permitan la colaboración o la competencia, sin embargo, las estrategias gamificadas que son dirigidas a la participación individual, pueden tornarse como barrera dado que no permite mecanismos ligados con el aprendizaje entre pares, ni tener una perspectiva de su desempeño respecto a la del compañero o contar con escenarios para el desarrollo de habilidades de trabajo colaborativo, así como el desarrollo de la motivación mediada por la competencia (de-Marcos et. al., 2014). 
En lo referente al Tiempo para el Aprendizaje, se establece que en las etapas de planeación o estructuración del diseño instruccional se debe contemplar la característica temporal, que indique la duración que debe estar expuesto el estudiante ante la estrategia gamificada para que se logre el procesamiento cognitivo y se apropie la adquisición tanto de conocimientos como de competencias. Algunos autores afirman que tener un tiempo muy restringido puede hacer que el aprendizaje no se presente, y más cuando el tiempo se ve limitado por las demás actividades que se deben desarrollar en la estructura curricular de la asignatura (Guin et al., 2012; Sánchez-Mena y Martí-Parreño, 2017).

Por último, el uso de Instrucciones sin relación con el contenido pedagógico en la gamificación, diluye la participación del estudiante empujándolo a desarrollar acciones únicamente relacionadas con el entretenimiento sin posibilitar el aprendizaje. La falta de instrucciones claras o de instrucciones significativas relacionadas con el proceso de gamificación universitaria se identifica como la barrera más sobresaliente a tener en cuenta para superar (Erhel y Jamet, 2013; Tan y Hew, 2016).

\section{Barrera Docente}

Esta categoría contiene seis barreras específicas a saber: Conocimiento sobre Diseño e Implementación de la Gamificación, Conocimiento en Recursos, Competencia Tecnológica del Docente, Actitud Docente, Percepciones sobre el Uso y Experiencia Previa. La mayor opinión encontrada se relaciona con la barrera específica Conocimiento sobre Diseño e Implementación de la Gamificación, que lógicamente afecta las otras barreras al relacionarla con la falta de conocimiento o habilidades ligadas a la creación de estrategias gamificadas, así como el desconocimiento sobre el concepto de gamificación como estrategia didáctica lo que genera un bajo interés en su aplicación en el aula (Wiggins, 2016). Situación similar ocurre con la falta de Conocimientos en Recursos, al propiciar estrategias gamificadas inapropiadas para la edad del estudiante o su nivel cognitivo (Canhoto y Murphy, 2016).

En cuanto a la Competencia Tecnológica Docente se identifica la presencia de competencias bajas en el uso de tecnologías que dinamicen el ejercicio educativo en el salón de clases debido a diferencias generacionales en las habilidades para el manejo de los dispositivos digitales (Hamzeh, et al., 2017). En lo relacionado con la Actitud Docente, se identifica que los maestros presentan una actitud negativa frente a la gamificación, debido a la incredulidad frente al impacto de los recursos gamificados en el aprendizaje (Castañeda, y Cho, 2016), o la creencia 
sobre la dificultad para aplicarlos en educación, disminuyendo su implementación en el aula (Martí, et al., 2018). En otros casos se argumenta por parte de los docentes su sentir sobre un aumento en la carga laboral relacionada con el diseño y creación de estrategias gamificadas, que en algunas ocasiones no es reconocida ni en tiempo ni en remuneración por las instituciones de educación superior (Ding, 2019), o casos donde los docentes presentan una actitud positiva pero poca habilidad en la implementación (Martí, et al., 2018).

De manera similar y relacionado con las percepciones sobre el uso de gamificación en el aula, algunos maestros consideran que la utilización de productos gamificados genera problemas en el manejo del tiempo respecto a los contenidos que se deben abordar en el aula, además de generar situaciones de distracción ligados con el manejo de las tecnologías en el salón (Martí et al., 2018). En otras ocasiones tienen la percepción de que los juegos son violentos y pueden propiciar patrones de agresión en la clase (Kirillov, et al., 2016).

Por último, se encuentra la Experiencia Previa. Al respecto se aduce que algunos docentes carecen de vivencias directas sobre el manejo de juegos digitales, lo que hace que puedan recurrir a estudiantes más diestros para apoyarse en la implementación de la gamificación en el aula. Sin embargo, se necesita de cierta experticia o cierto dominio de la estrategia para ser el orientador en la clase (Kopcha, et al., 2016).

\section{Barrera Estudiante}

En la barrera Estudiante se identificaron seis barreras específicas: Falta de Cultura en Trabajo Colaborativo, Motivación, Dificultad Tecnológica, Dificultad en el Aprendizaje, Experiencia Previa y Competencia. En cuanto a la Falta de Cultura en Trabajo Colaborativo se hallan casos de escaso interés del estudiante por participar de las actividades colaborativas propuestas para el alcance de los objetivos pedagógicos (Pérez et al., 2017). Igualmente, se destaca la influencia del estilo de aprendizaje del estudiante en la barrera específica motivación, ya que las estrategias didácticas gamificadas pueden no servir a todos, ni generar la misma emocionalidad al momento de la implementación (Domínguez et al., 2013) lo que permite resaltar la motivación como factor psicológico de impacto en el desempeño del juego y en el desempeño académico del estudiante. En cuanto a la motivación intrínseca de los estudiantes se argumenta que las personas con mayor competencia tecnológica como los nativos digitales, pueden presentar emocionalidad neutra respecto a los productos gamificados, versus aquellos que 
no lo son. Igualmente, aquellos estudiantes que presentan puntajes bajos en el desarrollo de la estrategia gamificada, suelen presentar baja motivación (Kyeswki y Krämer, 2018) o estudiantes que previamente han sido motivados pueden desmotivarse a lo largo del juego a causa de las expectativas generadas con anterioridad (van Roy y Zaman, 2018).

Por otra parte, el análisis permite inferir que para algunos estudiantes es difícil el manejo de la tecnología relacionada con la estrategia de gamificación, sobre todo para aquellos que no tienen acceso a los dispositivos tecnológicos o aquellos que pertenecen a culturas, donde tradicionalmente, no existe relación con las tecnologías o los juegos digitales (Villalustre y del Moral, 2015). En la categoría Dificultad en el Aprendizaje, algunos estudiantes presentan limitaciones para establecer la conexión entre el juego y los contenidos a aprender, debido a niveles atencionales bajos frente a estas estrategias (Subhash y Cudney, 2018) o al interés elevado en las características del juego más no en los elementos pedagógicos (Yildirim, 2017). Asimismo, la falta de experiencia, la carencia de conocimientos, o la ausencia de destrezas en el desarrollo de la estrategia gamificada, suscita que no se presente una adecuada implementación (Antonaci et al., 2015).

Como última categoría específica, se presenta el grado de rivalidad entre estudiantes como parte de la Competencia al interior de la estrategia gamificada; en este sentido, se ubica la dificultad para asumir de manera equilibrada la competencia y su relación con el aprendizaje, dado que algunos estudiantes o compiten o aprenden, este sentimiento de competencia genera sobrecarga cognitiva, fatiga y altos niveles de desgaste emocional (Rutledge et al., 2018).

\section{CONCLUSIONES}

La gamificación, como una forma de didáctica, procura la calidad académica y en muchos casos facilita la labor docente. Este documento resume la investigación realizada en la detección de barreras en la aplicación de didácticas gamificadas en el ámbito de la educación superior. La identificación de las barreras encontradas permite a investigadores, docentes e interesados entender y comprender, de mejor manera, los obstáculos que afectan el aprendizaje mediante gamificación, lo cual permite proponer mejoras que posibiliten la aplicación exitosa de estrategias gamificadas en el aula. Igualmente, este estudio permite inferir la importancia de la capacitación en competencias tecnológicas y digitales, pedagógicas y de diseño, con el fin de promover y cualificar en el maestro sus habilidades como desarrollador o creador 
de este tipo de estrategia didáctica. Es relevante comprender que los procesos de enseñanza-aprendizaje se adaptan a los cambios sociales, políticos y tecnológicos que pasan de las metodologías tradicionales a metodologías activas centradas en el estudiante, lo que involucra una actualización en conocimientos y un desarrollo en destrezas frente a los nuevos recursos emergentes.

En este sentido, es importante que las Instituciones de Educación Superior, procuren los recursos necesarios para lograr la implementación de estrategias gamificadas, así como el aseguramiento del soporte o mantenimiento necesario para llevar a cabo las actividades, garantizando con ello condiciones de calidad exigidas por los entes de control, relacionadas con la infraestructura para el ejercicio de la enseñanza - aprendizaje. El diseñar estrategias didácticas involucra una articulación con los contenidos pedagógicos, siendo de vital importancia el comprender las características propias de los estudiantes con el fin de lograr que los recursos didácticos sean pertinentes y acordes con los aspectos demográficos y socioculturales. Se infiere que la estética y el diseño de interfaz, deben motivar, pero no disminuir la atención del objeto de aprendizaje, ya que los diseños complejos fomentan la distracción y, en algunos casos, dificultan la resolución de los retos propuestos.

La identificación y categorización de las barreras que aquí se exponen ha sido un trabajo dinámico dada la propia movilidad de los procesos y los actores en educación superior, además de la variabilidad tecnológica y, lo principal, el impacto que este tipo de didáctica ha tenido en la pedagogía y en la transferencia de conocimiento. Este trabajo, no termina aquí. Se debe enriquecer con la identificación de otro tipo de barreras que emergerán posteriormente. Se destaca que las barreras identificadas pueden ser adaptadas como criterios de evaluación del proceso educativo, permitiendo mejorar las prácticas en el aula de tipo tradicional y digital, mediante la adecuada planeación e implementación de estrategias gamificadas.

Finalmente, como limitaciones se encontró el bajo número de artículos científicos específicos en cuanto a lo desarrollado por país o modalidad educativa, tampoco este trabajo investigativo se basó en la consulta de expertos en gamificación que permitieran evidenciar, de manera práctica, las barreras desde su experticia, ni se generó un análisis directo de contenidos digitales gamificados en educación superior, proceso que deberá llevarse a cabo como futuras líneas de investigación para poder contrastar con fuentes directas, a partir de entrevistas con expertos o en plataformas con contenido digital gamificado, con el fin de confirmar, adaptar y complementar las barreras aquí encontradas. 


\section{REFERENCIAS BIBLIOGRÁFICAS}

Acosta-Gonzaga, E., \& Walet, N. R. (2018). The role of attitudinal factors in mathematical on-line assessments: A study of undergraduate STEM students. Assessment $\mathbb{E}$ Evaluation in Higher Education, 43(5), 710-726. doi: https://doi.org/10.1080/02602938.2017.1401976

Antonaci, A., Dagnino, F. M., Ott, M., Bellotti, F., Berta, R., de Gloria, A., Lavagnino, E., Romero, M., Usart, M. \& Mayer, I. (2015). A gamified collaborative course in entrepreneurship: Focus on objectives and tools. Computers in Human Behavior, 51, 1276-1283. doi: https://doi.org/10.1016/j.chb.2014.11.082

Barr, M. (2017). Video games can develop graduate skills in higher education students: A randomised trial. Computers E Education, 113, 86-97. doi: https://doi.org/10.1016/j.compedu.2017.05.016

Bellotti, F., Berta, R., De Gloria, A., Lavagnino, E., Antonaci, A., Dagnino, F., Ott, M., Romero, M., Usart, M. \& Mayer, I. S. (2014). Serious games and the development of an entrepreneurial mindset in higher education engineering students. Entertainment Computing, 5(4), 357-366. doi: https://doi.org/10.1016/j.entcom.2014.07.003

Blevins, A.E., Kiscaden, E. \& Bengtson, J. (2017) Courting Apocalypse: Creating a ZombieThemed Evidence-Based Medicine Game. Medical Reference Services Quarterly, 36(4), 313. 322. doi: http://dx.doi.org/10.1080/02763869.2017.1369239

Bovermann, K., Weidlich, J. \& Bastiaens, T. (2018). Online learning readiness and attitudes towards gaming in gamified online learning - a mixed methods case study. International Journal of Educational Technology in Higher Education, 15(1), 1-17. doi: http://dx.doi.org/10.1186/s41239-018-0107-0

Cain, J. \& Piascik, P. (2015). Are serious games a good strategy for pharmacy education? American Journal of Pharmaceutical Education, 79(4), 1-6. doi: https://doi.org/10.5688/ajpe79447

Calabor, M. S., Mora, A., \& Moya, S. (2018). Adquisición de competencias a través de juegos serios en el área contable: un análisis empírico. Revista de Contabilidad, 21(1), 38-47. doi: https://doi.org/10.1016/j.rcsar.2016.11.001

Canhoto, A.I. \& Murphy, J. (2016). Learning from Simulation Design to Develop Better Experiential Learning Initiatives: An Integrative Approach. Journal of Marketing Education, 38(2), 98106. doi: https://doi.org/10.1177/0273475316643746

Domínguez, A., Sáenz, J., de-Marcos, L., Fernández, L., Pagés, C., \& Martínez, J. (2013). Gamifying learning experiences: Practical implications and outcomes. Computers $\mathcal{E}$ Education, 63, 380 392. doi: https://doi.org/10.1016/j.compedu.2012.12.020

Fotaris, P., Mastoras, T., Leinfellner, R. \& Rosunally, Y. (2016). Climbing up the leaderboard: An empirical study of applying gamification techniques to a computer programming class. Electronic Journal of e-Learning, 14(2), 94-110. Recuperado de:

https://www.researchgate.net/publication/293816223_Climbing_Up_the_Leaderboard_A n_Empirical_Study_of_Applying_Gamification_Techniques_to_a_Computer_Programming_Class [Fecha de consulta: enero 17 de 2019]. 
Gallegos, C., Tesar, A. J., Connor, K., \& Martz, K. (2017). The use of a game-based learning platform to engage nursing students: A descriptive, qualitative study. Nurse Education in Practice, 27, 101-106. doi: https://doi.org/10.1016/j.nepr.2017.08.019

Girard, C., Ecalle, J., \& Magnan, A. (2013). Serious games as new educational tools: How effective are they? A meta-analysis of recent studies. Journal of Computer Assisted Learning, 29 (3), $207-$ 219. doi: https://doi.org/10.1111/j.1365-2729.2012.00489.x_Goehle, G. (2013). Gamification and Web-based Homework. PRIMUS, 23(3), 234-246. doi: https://doi.org/10.1080/10511970.2012.736451

Gómez-Urquiza, J.L., Gómez-Salgado, J., Albendín-García, L., Correa-Rodríguez, M., GonzálezJiménez, E. \& Cañadas-De la Fuente, G.A. (2019). The impact on nursing students' opinions and motivation of using a "Nursing Escape Room" as a teaching game: A descriptive study. Nurse Education Today, 72, 73-76. doi: http://dx.doi.org/10.1016/j.nedt.2018.10.018

Mora, A., Riera, D., González, C. \& Arnedo-Moreno, J. (2017). Gamification: a systematic review of design frameworks. Journal of Computing in Higher Education, 29(3), 516-548. doi: https://doi.org/10.1007/s12528-017-9150-4

Nebel, S., Schneider, S., Beege, M., \& Rey, G. D. (2017). Leaderboards within educational videogames: The impact of difficulty, effort and gameplay. Computers $\mathscr{E}$ Education, (113), 2841. doi: https://doi.org/10.1016/j.compedu.2017.05.011

Olsson, M., Mozelius, P.\& Collin, J. (2015). Visualisation and gamification of e-Learning and programming education. Electronic Journal of e-Learning, 13(6), 441-454. Recuperado de: https://eric.ed.gov/?id=EJ1087309 [Fecha de consulta: enero 21 de 2019].

Poondej, C. \& Lerdpornkulrat, T. (2016). The development of gamified learning activities to increase student engagement in learning. Australian Educational Computing, 31(2), 1-16. Recuperado de: https://www.semanticscholar.org/paper/The-development-of-gamifiedlearning-activities-to-Poondej-Lerdpornkulrat/d6921ac0724ee4554a9f2e0de566521 c8a311f21 [Fecha de consulta: enero 22 de 2019].

Reid, A., Paster, D., \& Abramovich, S. (2015). Digital badges in undergraduate composition courses: Effects on intrinsic motivation. Journal of Computers in Education, 2(4), 377-398. doi: https://doi.org/10.1007/s40692-015-0042-1

Rutledge, C., Walsh, C.M., Swinger, N., Auerbach, M., Castro, D., Dewan, M., Khattab, M., Rake, A., Harwayne-Gidansky, I., Raymond, T.T., Maa, T. \& Chang, T.P. (2018). Gamification in action: Theoretical and practical considerations for medical educators. Academic Medicine, 93 (7), 1014-1020. doi: http://dx.doi.org/10.1097/ACM.0000000000002183

Sánchez-Mena, A. \& Martí-Parreño, J. (2017). Drivers and barriers to adopting gamification: Teachers' perspectives. Electronic Journal of e-Learning, 15(5), 434-443. Recuperado de: https://eric.ed.gov/?id=EJ1157970 [Fecha de consulta: enero 22 de 2019].

Santhanam, R., Liu, D. \& Shen, W.-C.M. (2016). Gamification of technology-mediated training: Not all competitions are the same. Information Systems Research, 27(2), 453-465. doi: https://doi.org/10.1287/isre.2016.0630

Snyder, E. \& Hartig, J.R. (2013). Gamification of board review: a residency curricular innovation. MedicalEducation, 47(5), 524-525. doi: http://dx.doi.org/10.1111/medu.12190 
Soflano, M., Connolly, T. M., \& Hainey, T. (2015). An application of adaptive games-based learning based on learning style to teach SQL. Computers $\mathcal{E}$ Education, 86, 192-211. doi: https://doi.org/10.1016/j.compedu.2015.03.015

Song, D., Ju, P. \& Xu, H. (2017). Engaged cohorts: Can gamification engage all college students in class? Eurasia Journal of Mathematics, Science and Technology Education, 13(7), 3723-3734. doi: http://dx.doi.org/10.12973/eurasia.2017.00755a

Subhash, S., \& Cudney, E. A. (2018). Gamified learning in higher education: A systematic review of the literature. Computers in Human Behavior, 87, 192-206. doi: https://doi.org/10.1016/j.chb.2018.05.028

Tan, L. (2018). Meaningful gamification and students' motivation: A strategy for scaffolding reading material. Online Learning, 22(2), 1-16. doi: https://doi.org/10.24059/olj.v22i2.1167

Tan, M. \& Hew, K.F. (2016). Incorporating meaningful gamification in a blended learning research methods class: Examining student learning, engagement, and affective outcomes. Australasian Journal of Educational Technology, 32 (5), 19-34. doi: https://doi.org/10.14742/ajet.2232

Tenório, T., Bittencourt, I. I., Isotani, S., Pedro, A., \& Ospina, P. (2016). A gamified peer assessment model for on-line learning environments in a competitive context. Computers in Human Behavior, 64, 247-263. doi: https://doi.org/10.1016/j.chb.2016.06.049

Torres, A., Romero, L., Pérez, M. \& Björk, S. (2016). Modelo Teórico Integrado de Gamificación en Ambientes E-Learning (E-MIGA). Revista Complutense de Educación, 29(1), 129-145. doi: https://doi.org/10.5209/RCED.52117

Van Roy, R., \& Zaman, B. (2018). Need-supporting gamification in education: An assessment of motivational effects over time. Computers EF Education, 127, 283-297. doi: https://doi.org/10.1016/j.compedu.2018.08.018 